\title{
Is gene therapy the answer for prostate cancer?
}

\author{
P Patel $^{1}$, D Ashdown ${ }^{1} \& \mathrm{~N}$ James $^{1 *}$ \\ ${ }^{1}$ Cancer Research UK Institute for Cancer Studies, University of Birmingham, \\ Edgbaston, Birmingham, UK
}

\begin{abstract}
Prostate cancer is the third most common cancer, accounting for one in $\mathbf{1 0}$ cancer diagnoses in men worldwide during 2000. Despite this high burden of morbidity, there is a lack of curative treatments for locally advanced and metastatic disease. Good anatomical accessibility of the prostate combined with substantial molecular understanding of the disease makes prostate cancer an attractive target for gene therapy. Considerable progress has been made in the development of suitable gene transfer vectors and prostate-targeting strategies. Therapeutic approaches being explored fall into two broad categories: corrective and cytoreductive/cytolytic. There are currently 63 prostate cancer gene therapy clinical trials based on these approaches registered in the United States and United Kingdom. Although significant hurdles remain to be overcome, early clinical trial results are encouraging, suggesting that gene therapy may become an important treatment option for prostate cancer.

Prostate Cancer and Prostatic Diseases (2004) 7, S14-S19. doi:10.1038/sj.pcan.4500743
\end{abstract}

Keywords: prostate neoplasms; gene therapy; genetic vectors; clinical trials; drugdelivery systems

\section{Introduction}

Prostate cancer is the third most common cancer, accounting for one in 10 cancer diagnoses in men worldwide during 2000. ${ }^{1}$ Patients initially presenting with localised prostate cancer can be controlled effectively with standard therapies in the short term. However, a proportion of patients ultimately relapse, and there is currently no curative therapy for locally advanced or metastatic disease in the hormonerefractory setting. ${ }^{2}$ In addition, a window of opportunity exists between the development of local treatment failure and the appearance of metastases, making direct local delivery of therapy a potentially viable treatment option. Therefore, successful gene therapy for prostate cancer has the potential to meet a substantial clinical need.

A number of factors make prostate cancer a particularly attractive target for gene therapy, including the anatomy of the organ, the natural history of the disease and the advanced knowledge of the molecular basis of prostate cancer. ${ }^{3}$ The prostate is easily accessible for localised administration of treatment, and a range of

*Correspondence: N James, Cancer Research UK Institute for Cancer Studies, University of Birmingham, Edgbaston, Birmingham B15 2TT, UK.

E-mail: jamesnd@cancer.bham.ac.uk techniques, including digital rectal examination, ultrasound, magnetic resonance imaging, and serum prostatespecific antigen (PSA) testing, are available for close monitoring of therapy. Prostate cancer has a long preclinical latency period, providing the opportunity for implementation of strategies to prevent disease progression in addition to the possibility of treating advanced and metastatic disease. Considerable progress has been made in elucidating the genetic basis for the molecular progression of prostate cancer, providing rational targets for corrective strategies. ${ }^{4}$ Several wellstudied markers of normal or cancerous prostate tissue have been described, including PSA, prostate-specific membrane antigen (PSMA), mucin (MUC-2), and human glandular kallikrein 2 (hK2). ${ }^{2}$ Moreover, about 500 prostate-specific expressed sequence tags have been identified so far. ${ }^{5}$ The products of these prostate-specific or cancer-specific genes provide targets for immunotherapy, and a practical means of directing and restricting therapy to the prostate.

The prostate is a nonessential organ, so cytotoxic effects on prostate cells neighbouring the tumour should not be prohibitive to the development of a treatment method; indeed, ablation of noncancerous prostate tissue may also provide relief from coexisting benign prostatic hyperplasia. ${ }^{6}$ The possibility of designing treatments aimed at the entire prostate, rather than the tumour itself, broadens the range of targeting strategies available for 
gene therapy. Prostate cancer is relatively slow-growing, with as few as $1.3 \%$ of cells entering the S-phase of cell division per day. $^{7}$ Therefore, many tumours may be resistant to S-phase-dependent cytotoxic strategies, and an ability to transduce nondividing prostate cells may be an important prerequisite for in vivo gene therapy.

\section{Tools for gene therapy}

\section{Gene transfer systems}

The choice of vector is a crucial aspect of any gene therapy strategy, and will have a significant influence on treatment success. Several different viral vectors have been used for gene therapy of prostate cancer, each of which has advantages and limitations (Table 1). For example, vaccinia virus can be produced to high titres in vitro, provides relatively long-lived gene expression and is able to package a large amount of foreign DNA, but has low gene transfer efficiency and only infects dividing cells. Adenovirus, on the other hand, has a high transduction efficiency and infects nondividing cells, potentially making it more useful for treatment of prostate cancer. To date, most trials have used a replication-defective adenovirus, in which two genes crucial for the generation of new virus particles, E1 and E3, have been deleted, although trials of conditionally replicating vectors have also been undertaken. However, the maximum permissible transgene size for an E1-, E3deleted replication-defective adenovirus is 8 kilobases $(\mathrm{kb})$. Despite this disadvantage, adenovirus has been the most frequently used viral vector for prostate cancer gene therapy. In addition to viral vectors, nonviral gene delivery systems, including naked DNA, liposomes, and DNA-protein complexes, are being used in several immunotherapy trials for prostate cancer. ${ }^{9}$ However, the low transfection efficiency and limited expression time with these delivery methods have generally limited their use to ex vivo approaches.

\section{Vector targeting}

A significant potential advantage of gene therapy is precise restriction of the treatment to the cancer, sparing the rest of the body from detrimental side effects. There are several levels at which in vivo specificity can be attained. One strategy is to target the vector to the cells of interest. This can be achieved either by direct injection into the prostate, or by using a vector which has a specific tropism for prostate or tumour tissue. ${ }^{8}$ Direct injection is an appealing method for targeting gene therapy vectors to the prostate, because of its accessibility, but has the disadvantage that metastases cannot be treated. ${ }^{8}$ There are no known naturally prostate-tropic viruses. ${ }^{10}$ However, some progress has been made in the alteration or enhancement of viral tropism for specific tissues or tumours. ${ }^{10}$ Future treatments may involve chemically retargeting viruses by polymer coating followed by addition of a targeting ligand, or genetic modification to alter receptor tropism (eg by simultaneously preventing binding to the widely distributed coxsackie-adenovirus receptor (CAR) while introducing ligands specific for other cell surface receptors such as human epidermal growth factor receptor 2 (HER2) or gonadotrophin-releasing hormone receptor). ${ }^{11}$

An alternative gene therapy targeting strategy, which has attracted maximum interest so far, is restricting the effect of the transgene to the prostate or prostate cancer cell population. ${ }^{10}$ Specificity of gene expression can be achieved by placing the transgene under the control of prostate- or tumour-specific regulatory elements. The wide range of known prostate-specific genes makes this an attractive strategy for prostate cancer. A potential drawback of this approach is that the transgene may not be activated in poorly differentiated cancer cells, which may also be resistant to other forms of treatment, for example, the androgen-independent cells in advanced prostate cancer. ${ }^{0}$ Furthermore, the level of transgene expression will be dependent on the promoter strength and activity in the target cell, and may not be sufficient to achieve the desired therapeutic effect. However, considerable progress has been made in developing prostate-specific promoter/enhancer constructs, which can achieve high levels of specific expression. $^{12}$

\section{Therapeutic strategies}

The therapeutic strategies employed in gene therapy fall into two main categories (Table 2). The first is corrective therapy, which aims to counteract the effect of absent or abnormal gene expression. The genes targeted in corrective gene therapy are generally either tumour suppressor genes or oncogenes. The second category is

Table 1 Characteristics of vectors utilised in current prostate cancer gene therapy trials ${ }^{2,6}$

\begin{tabular}{|c|c|c|c|c|}
\hline Characteristic & Retroviruses & Adenovirus & Adeno-associated virus & Vaccinia/fowlpox virus \\
\hline Size limit of transgene $(\mathrm{kb})$ & $5-8$ & 8 & $2-4.5$ & 25 \\
\hline Transduction efficiency & Low & High & High & Low \\
\hline Infection of nondividing cells & $\begin{array}{l}\text { No (oncoviruses) } \\
\text { Yes (lentiviruses, } \\
\text { spumaviruses) }\end{array}$ & Yes & Yes & No \\
\hline Genome integration & Yes & No & Yes (site-specific) & No \\
\hline Persistence of gene expression & Stable & $\begin{array}{l}\text { Transient unless } \\
\text { replication-competent }\end{array}$ & Stable & $\sim 1$ month \\
\hline Induces inflammatory/immune response & No & Yes & No & Yes \\
\hline High viral titres achievable in vitro & No & Yes & Yes & Yes \\
\hline
\end{tabular}


Table 2 Therapeutic strategies for prostate cancer gene therapy

\begin{tabular}{|c|c|}
\hline Therapeutic strategy & Description \\
\hline \multicolumn{2}{|l|}{ Corrective } \\
\hline Tumour suppressor gene replacement & $\begin{array}{l}\text { Introduction of a wild-type copy of a tumour suppressor gene to correct aberrant or absent } \\
\text { expression }\end{array}$ \\
\hline Oncogene suppression & Introduction of antisense RNA or ribozyme to inhibit expression of an oncogene \\
\hline \multicolumn{2}{|l|}{ Cytoreductive/cytolytic } \\
\hline Immunotherapy & Range of methods designed to stimulate immune destruction of tumour cells \\
\hline Oncolytic therapy & Virus is engineered to replicate only in tumour cells, resulting in their specific destruction \\
\hline Enzyme/prodrug therapy & $\begin{array}{l}\text { An enzyme encoded by a tumour-targeted vector converts a harmless prodrug into a cytotoxic } \\
\text { compound }\end{array}$ \\
\hline
\end{tabular}

cytoreductive or cytolytic therapy, which aims to destroy the tumour cells.

\section{Corrective therapy}

Tumour suppressor gene replacement A number of genes that are altered or deleted in a high proportion of prostate cancer clinical specimens or cell lines have been identified as targets for corrective therapy and, in several cases, validated in experimental models. For example, the tumour suppressor gene p53 is mutated in many prostate cancer cell lines and clinical specimens. ${ }^{13}$ Replacement of this gene can suppress growth of prostate cancer cell lines both in vitro and in animals, and of primary cultures from radical prostatectomy specimens. ${ }^{14,15}$ Similarly, loss of expression of another tumour suppressor gene, $p 16$, in locally advanced prostate cancer is associated with a significantly increased risk of local progression, metastasis and reduced survival. ${ }^{16}$ Injection of adenovirus containing the wildtype $p 16$ gene into prostate cancer cell tumours in mice resulted in reduced tumour size and longer survival compared with mice treated with control virus. ${ }^{17}$ Surprisingly, although it would be logical to expect that the effect of tumour suppressor gene replacement would be restricted to transfected cells, a strong 'bystander' effect has been noted, in which neighbouring cells are also killed following therapy. ${ }^{18}$

Oncogene suppression Oncogene suppression is most often achieved by expression of either antisense oligonucleotides, which bind in a sequence-specific manner to mRNA and prevent its translation, or ribozymes (catalytically active RNA molecules that cleave specific mRNA sequences). ${ }^{19}$ Several oncogenes (eg c-myc, bcl-2) have been shown to be overexpressed in prostate cancer: treatment with a retrovirus expressing antisense c-myc resulted in suppression of tumour growth in a model of human prostate cancer. ${ }^{20}$.

A drawback to corrective therapy is that multiple mutations in different pathways are frequently present in prostate cancer, which suggests that developing an effective corrective strategy will require a multifactorial approach. ${ }^{21}$ Furthermore, for this type of therapy to be successful, all the tumour cells must be corrected, requiring high transduction and expression efficiencies that may well be beyond the capabilities of today's gene transfer systems. ${ }^{2}$ Therefore, this strategy may ultimately be most appropriate for use in combination with other therapeutic approaches. ${ }^{8}$

\section{Cytoreductive/cytolytic therapy}

Immunotherapy The most widely investigated cytoreductive/cytolytic approach is immunotherapy, in which the host immune system is stimulated to act against tumour cells. Several immunotherapeutic approaches for prostate cancer gene therapy have been described. Many tumours are defective in antigen presentation via major histocompatibility complex (MHC) class I, and therefore avoid the immune response. By transforming tumour cells with genes encoding cytokines, antigen presentation and immune stimulation by these cells is enhanced. ${ }^{2}$ An alternative is to transduce dendritic cells or other cells (eg muscle) with tumour antigens (anticancer vaccination). A Phase I clinical trial using autologous $\mathrm{T}$ lymphocytes (adoptive T-cell therapy) stimulated in vitro with synthetic peptides presented by dendritic cells is currently ongoing in the UK. Theoretically, immune responses elicited against tumour cells should act against metastases at distant sites in addition to the primary tumour. ${ }^{21} \mathrm{~A}$ disadvantage of some immunotherapy protocols is that isolation and ex vivo cultivation of host tumour or immune cells is often involved, which is expensive and technically difficult. ${ }^{2}$

Enzyme/prodrug therapy A further approach is enzyme/ prodrug therapy, in which a vector is targeted to tumour cells to produce an exogenous enzyme. Concurrently, a harmless prodrug is administered - the enzyme converts the prodrug into its cytotoxic form within the tumour cells. The cytotoxic effect of enzyme/prodrug therapy occurs only upon administration of a prodrug, allowing immediate cessation in the case of serious adverse effects of therapy. ${ }^{6}$ Several prodrugs are already in clinical use, simplifying the process of obtaining approval for trials. However, precise restriction of transgene expression to the tumour cells, either by vector targeting or gene regulation, is essential to minimise concurrent damage to healthy tissue. A substantial bystander effect has been observed for suicide therapy, with cytotoxicity rates of 100-1000 times those predicted by gene transfer rates alone, which may help to compensate for low gene transfer and expression levels. ${ }^{22}$

The most common enzyme/prodrug system used to date as gene therapy for prostate cancer is the herpes simplex virus (HSV) thymidine kinase (TK) in combination with the prodrug ganciclovir or acyclovir. The HSV TK enzyme phosphorylates the prodrug, which is then available for further phosphorylation by cellular TK. The resulting nucleoside triphosphate can be incorporated 
into nascent DNA during synthesis, leading to chain termination, which inhibits DNA replication and thus cell division. HSV TK plus acyclovir significantly inhibited tumour growth in an experimental human prostate cancer mouse model. ${ }^{23}$ A disadvantage of this system is that it only kills rapidly dividing cells, so other enzyme/prodrug combinations may ultimately prove to be more clinically useful for treatment of prostate cancer. One promising alternative is the nitroreductase/CB1954 system, in which the bacterial enzyme nitroreductase activates the prodrug CB1954 to form a highly potent DNA alkylating agent. ${ }^{24}$ The lack of cell-cycle specificity, as well as a moderate bystander effect, are advantages of this enzyme/prodrug combination. ${ }^{25}$

Oncolytic therapy Another cytoreductive/cytolytic technique is oncolytic therapy, in which a virus is designed to preferentially replicate within tumour cells, resulting in their death. This is achieved by placing a gene essential for viral replication under the control of a tumourspecific promoter, to form a conditionally replicating virus. ${ }^{2}$ The first such virus to be tested in humans was dl1520 (Onyx-015), an adenovirus with a deletion of a gene encoding a p53-inhibitory protein, E1B-55 kD. ${ }^{26}$ As a result of its inability to inactivate $p 53$, this virus is selective for tumours that have already lost $p 53$ function. As viable virus particles are generated during infection of cells with replication-conditional virus, in vivo amplification of the therapeutic effect can occur through further rounds of infection. ${ }^{8}$ Therefore, oncolytic viruses have the potential to infect and destroy a much higher proportion of tumour cells, including metastases, than the replication-defective vectors used in other gene therapy approaches. ${ }^{2}$ However, oncolytic therapy requires careful targeting of virus replication to the desired cell type to avoid toxicity. Some success with this approach has been demonstrated using the PSA enhancer/promoter to direct lytic replication of adenovirus in an animal model of human prostate cancer. ${ }^{27}$

\section{Clinical trials}

Gene therapy was first administered to a prostate cancer patient in $1994 .^{9}$ After $10 \mathrm{y}$, there have still been no Phase III trials, nor any large statistically powered Phase II trials, which can demonstrate clinical benefits from prostate cancer gene therapy. However, the available data have demonstrated the potential good safety and tolerability, and feasibility of some methods, and there is considerable continued research activity. The majority of clinical trials for gene therapy in prostate cancer have taken place in the US. By February 2004, 61 trials were registered on the Office of Biologic Activities Human Gene Transfer Protocol List (Table 3), of which 43 were open, 13 closed and one completed. ${ }^{9}$ Three trials were never initiated and one is still in submission. In contrast, there have been only two such trials in the UK. ${ }^{28}$

Most gene therapy trials for prostate cancer have used immunotherapeutic approaches. A detailed discussion of immunotherapy trials for prostate cancer gene therapy is beyond the scope of this publication, and the topic has been comprehensively covered elsewhere. ${ }^{29}$ Here we review recent developments in trials focusing on a direct tumour attack strategy, of which 23 are in progress in the
Is gene therapy the answer for prostate cancer?

P Patel et al

Table 3 Prostate cancer gene therapy trials in the $\mathrm{US}^{9}$ and $\mathrm{UK}^{28}$

\begin{tabular}{|c|c|c|}
\hline Category & Gene/promoter & $\begin{array}{l}\text { Number } \\
\text { of trials }\end{array}$ \\
\hline \multicolumn{3}{|l|}{ Corrective } \\
\hline \multirow{3}{*}{$\begin{array}{l}\text { Tumour } \\
\text { suppressor gene }\end{array}$} & p53 & 3 \\
\hline & & \\
\hline & $p 16$ & 1 \\
\hline Oncogene & $\mathrm{c}-m y c$ & 1 \\
\hline \multicolumn{3}{|l|}{$\begin{array}{l}\text { Cytoreductive/ } \\
\text { cytolytic }\end{array}$} \\
\hline \multirow[t]{13}{*}{ Immunotherapy } & PSA & 10 \\
\hline & GM-CSF & 6 \\
\hline & IL-2 & 4 \\
\hline & $\begin{array}{l}\text { Human telomerase reverse } \\
\text { transcriptase }\end{array}$ & 3 \\
\hline & PSA/CD80 & 3 \\
\hline & IL-12 & 2 \\
\hline & MUC-1/IL-2 & 2 \\
\hline & PSA/CD80/ICAM-1/LFA-3 & 2 \\
\hline & IFN- $\beta$ & 1 \\
\hline & PSA/CD80/ICAM-1/LFA-3/GM-CSF & 1 \\
\hline & PSMA & 1 \\
\hline & Total tumour RNA & 1 \\
\hline & TRAIL & 1 \\
\hline \multirow{4}{*}{$\begin{array}{l}\text { Enzyme/ } \\
\text { prodrug }\end{array}$} & HSV TK & 5 \\
\hline & HSV TK/cytosine deaminase & 5 \\
\hline & Cytochrome P450 & $1^{\mathrm{a}}$ \\
\hline & Nitroreductase/CB1954 & $1^{\mathrm{a}}$ \\
\hline \multirow[t]{2}{*}{ Oncolytic } & PSA & 4 \\
\hline & Osteocalcin & 1 \\
\hline $\begin{array}{l}\text { Gene-directed } \\
\text { radioisotope } \\
\text { therapy }\end{array}$ & Sodium/iodide symporter & 1 \\
\hline \multicolumn{3}{|l|}{${ }^{\mathrm{a}} \mathrm{UK}$-based trials } \\
\hline \multicolumn{3}{|c|}{$\begin{array}{l}\text { Trials that are open, closed, completed, under review, or in submission are } \\
\text { included. Trials that are closed having never been initiated are excluded. GM- } \\
\text { CSF, granulocyte-macrophage colony-stimulating factor; HSV TK, herpes } \\
\text { simplex virus thymidine kinase; ICAM-1, intercellular adhesion molecule 1; } \\
\text { IFN- } \beta \text {, interferon- } \beta \text {; IL, interleukin; LFA-3, leucocyte function antigen } 3 \text {; } \\
\text { MUC- } 1 \text {, mucin-1; PSA, prostate-specific antigen; PSMA, prostate-specific } \\
\text { membrane antigen; RNA, ribonucleic acid; TRAIL, tumour necrosis factor- } \\
\text { related apoptosis-inducing ligand. }\end{array}$} \\
\hline
\end{tabular}

US and UK (Table 3). The vast majority of these trials employ adenovirus, with two studies involving retroviral vectors.

\section{Enzyme/prodrug therapy}

The major focus of attention in tumour-directed prostate cancer trials has been on enzyme/prodrug systems, with most studies using HSV TK alone or in combination with cytosine deaminase. Two studies of intraprostatic injection of adenovirus expressing HSV TK followed by systemic administration of ganciclovir reported only mild and self-limiting toxicity. ${ }^{30,31}$ Encouragingly, preliminary results in one of these studies indicated that 12 out of 28 patients experienced a $44 \%$ mean decrease in PSA levels, ${ }^{30}$ and in the other, three of 18 patients experienced a decrease in PSA levels of $>50 \%$, lasting from 6 weeks to $1 \mathrm{y}^{31}$ In a third trial, adenovirus expressing HSV TK under the control of the osteocalcin 
promoter was directly injected into metastatic lesions or postsurgical recurrences of prostate cancer. ${ }^{32}$ Effective targeting of TK expression was achieved, as demonstrated by lack of hepatic toxicity following valacyclovir administration, even though viral particles were detected in the serum.

Our group is conducting a Phase I trial, involving direct intraprostatic injection of a replication-defective adenovirus, designated CTL102, which expresses nitroreductase under the control of the CMV immediate early promoter. $^{33}$ In the first phase of the trial, patients awaiting radical prostatectomy were injected with escalating doses of CTL102 2 days prior to surgery, under transrectal ultrasound (TRUS) monitoring. Preliminary results have demonstrated favourable safety and tolerability profiles, and a similar result using the same vector was seen in a sister trial performed on patients with resectable liver cancers. ${ }^{34}$ The second phase of the trial, in which inoperable patients will receive the prodrug CB1954, is now open for recruitment. This section of the trial is designed to further examine safety and tolerability, and gives the first opportunity to detect antitumour activity.

\section{Oncolytic therapy}

Clinical trial results with oncolytic therapy are also beginning to emerge: the first Phase I study to be reported involved intraprostatic delivery of a replicationconditional adenovirus in which the E1A gene was under the control of PSA promoter/enhancer elements. A $>50 \%$ reduction in serum PSA was achieved in five out of 20 patients in this dose-escalation study: all responders were in the two highest dosage groups. ${ }^{35}$ Only mild adverse events were observed, indicating that the PSA promoter/enhancer restriction is stringent enough to limit replication of the virus in other cell types.

\section{Future directions}

Although gene therapy holds the promise of effective treatment for prostate cancer, significant obstacles must be overcome before it can reach the clinic. One issue is target validation-more progress needs to be made in confirming a sound scientific basis for the choice of each gene therapy target. The development of improved gene therapy vectors is also likely to be an important focus of research. There is a clear need to develop synthetic or highly modified viral vectors that combine most advantages of current gene transfer systems while minimising their disadvantages. These should facilitate precise and efficient delivery of transgenes of increased size to a specific target cell population, in the absence of a deleterious inflammatory or immune response. We are still far from generating an 'ideal' vector, but feedback from clinical trials will help in the development of more refined gene delivery systems. The third area in which progress needs to be made is in translating the principles of rational target design and vector development into clinically relevant effects and benefits. Although trials to date have shown that gene therapy for prostate cancer can be well tolerated, little progress has been made in demonstrating a clinical effect of any treatment. The clinical trials so far have been promising, but there is a need for Phase III trials powered to demonstrate therapeutic benefits. Only when these results begin to accumulate will we get a real idea of the likely impact that gene therapy may have on prostate cancer treatment.

Prostate cancer management currently relies on the integration of a number of treatment modalities, including surgery, radiotherapy, hormonal therapy, and chemotherapy. For example, one approach that has shown promise in the laboratory is the combination of gene therapy with radiotherapy. Radiation may improve transduction efficiency and transgene integration, while drugs that act on DNA synthesis (introduced via enzyme/prodrug gene therapy strategies) may increase the susceptibility of the tumour to the cytotoxic effects of radiotherapy. ${ }^{36}$ Preclinical studies have shown that multimodal treatment may have a synergistic effect on treatment efficacy when compared with the effects of either treatment modality alone, in an animal prostate cancer model. ${ }^{37}$ A clinical trial is in progress to examine the combination of HSV TK suicide therapy with conventional radiotherapy. ${ }^{36}$ It is feasible that a role in localised therapy may be established in the relatively near future. Substantial obstacles to successful therapy for metastatic disease remain: in particular, the challenge of directing the virus to widely distributed targets while avoiding the problems of infection, doselimiting toxicity to normal tissues, and sequestration of virus by the immune system. In conclusion, it is likely that, rather than replacing these established therapies, the future for gene therapy lies mainly in multimodal treatment.

\section{References}

1 Ferlay J, Bray F, Pisani P, Parkin DM. GLOBOCAN 2000: Cancer Incidence, Mortality and Prevalence Worldwide, Version 1.0. IARC CancerBase No. 5. IARC Press: Lyon, 2001.

2 Mabjeesh NJ, Zhong H, Simons JW. Gene therapy of prostate cancer: current and future directions. Endocr Relat Cancer 2002; 9: 115-139.

3 Steiner MS, Gingrich JR, Chauhan RD. Prostate cancer gene therapy. Surg Oncol Clin N Am 2002; 11: 607-620.

4 Nicholson B, Theodorescu D. Molecular therapeutics in prostate cancer. Histol Histopathol 2003; 18: 275-298.

5 Nelson PS et al. The prostate expression database (PEDB): status and enhancements in 2000. Nucleic Acids Res 2000; 28: 212-213.

6 Gardner TA, Sloan J, Raikwar SP, Kao C. Prostate cancer gene therapy: past experiences and future promise. Cancer Metastasis Rev 2002; 21: 137-145.

7 Berges RR et al. Cell proliferation, DNA repair, and $p 53$ function are not required for programmed death of prostatic glandular cells induced by androgen ablation. Proc Natl Acad Sci USA 1993; 90: 8910-8914.

$8 \mathrm{Lu} \mathrm{Y}$. Viral based gene therapy for prostate cancer. Curr Gene Ther 2001; 1: 183-200.

9 Office of Biotechnology Activities, National Institutes of Health. Human Gene Transfer Protocols. 3 February 2004. Available from: http://www4.od.nih.gov/oba/rac/PROTOCOL.pdf (accessed 10 May 2004).

10 Bangma $\mathrm{CH}$. Targeting of adenoviral vectors for gene therapy of prostate cancer. Prostate Cancer Prostatic Dis 2000; 3: 308-312.

11 Seymour LW et al. Adenovirus retargeting and systemic delivery. Ernst Schering Res Found Workshop 2003; 43: 107-114. 
12 Latham JP, Searle PF, Mautner V, James ND. Prostate-specific antigen promoter/enhancer driven gene therapy for prostate cancer: construction and testing of a tissue-specific adenovirus vector. Cancer Res 2000; 60: 334-341.

13 Heidenberg $\mathrm{HB}$ et al. Alteration of the tumor suppressor gene p53 in a high fraction of hormone refractory prostate cancer. J Urol 1995; 154: 414-421.

$14 \mathrm{Ko}$ SC et al. Molecular therapy with recombinant $p 53$ adenovirus in an androgen-independent, metastatic human prostate cancer model. Hum Gene Ther 1996; 7: 1683-1691.

15 Asgari $\mathrm{K}$ et al. Inhibition of the growth of pre-established subcutaneous tumor nodules of human prostate cancer cells by single injection of the recombinant adenovirus $p 53$ expression vector. Int J Cancer 1997; 71: 377-382.

16 Chakravarti A et al. Loss of p16 expression is of prognostic significance in locally advanced prostate cancer: an analysis from the Radiation Therapy Oncology Group protocol 86-10. J Clin Oncol 2003; 21: 3328-3334.

17 Steiner MS et al. Adenoviral vector containing wild-type p16 suppresses prostate cancer growth and prolongs survival by inducing cell senescence. Cancer Gene Ther 2000; 7: 360-372.

18 Fujiwara T, Kataoka M, Tanaka N. Adenovirus-mediated p53 gene therapy for human cancer. Mol Urol 2000; 4: 51-54.

19 Ast G. Drug-targeting strategies for prostate cancer. Curr Pharm Des 2003; 9: 455-466.

20 Steiner MS, Anthony CT, Lu Y, Holt JT. Antisense c-myc retroviral vector suppresses established human prostate cancer. Hum Gene Ther 1998; 9: 747-755.

21 Harrington KJ et al. Gene therapy for prostate cancer: current status and future prospects. J Urol 2001; 166: 1220-1233.

22 Kerr DJ, Young LS, Searle PF, McNeish IA. Gene directed enzyme prodrug therapy for cancer. Adv Drug Deliv Rev 1997; 26: 173-184.

23 Cheon J et al. Adenovirus-mediated suicide-gene therapy using the herpes simplex virus thymidine kinase gene in cell and animal models of human prostate cancer: changes in tumour cell proliferative activity. BJU Int 2000; 85: 759-766.

24 Denny WA. Nitroreductase-based GDEPT. Curr Pharm Des 2002; 8: 1349-1361.

25 Bridgewater JA et al. The bystander effect of the nitroreductase/ CB1954 enzyme/prodrug system is due to a cell-permeable metabolite. Hum Gene Ther 1997; 8: 709-717.

26 Bischoff JR et al. An adenovirus mutant that replicates selectively in p53-deficient human tumor cells. Science 1996; 274: 373-376.

27 Rodriguez $\mathrm{R}$ et al. Prostate attenuated replication competent adenovirus (ARCA) CN706: a selective cytotoxic for prostatespecific antigen-positive prostate cancer cells. Cancer Res 1997; 57: 2559-2563.

28 Gene Therapy Advisory Committee, Department of Health Latest UK gene therapy research 1993-2003. 15 April 2004 Available from: http://www.advisorybodies.doh.gov.uk/genetics/gtac/genetherapyresearch1993to2003.pdf (accessed 10 May 2004).

29 Kaminski JM et al. Immunotherapy and prostate cancer. Cancer Treat Rev 2003; 29: 199-209.

30 Shalev $\mathrm{M}$ et al. Suicide gene therapy toxicity after multiple and repeat injections in patients with localized prostate cancer. J Urol 2000; 163: 1747-1750.

31 Herman JR et al. In situ gene therapy for adenocarcinoma of the prostate: a phase I clinical trial. Hum Gene Ther 1999; 10: 1239-1249.

32 Kubo $\mathrm{H}$ et al. Phase I dose escalation clinical trial of adenovirus vector carrying osteocalcin promoter-driven herpes simplex virus thymidine kinase in localized and metastatic hormonerefractory prostate cancer. Hum Gene Ther 2003; 14: 227-241.

33 James ND et al. A biodistribution study of adenovirus-delivered nitroreductase (Ad-NTR) in patients with operable prostate cancer. Proc Am Soc Clin Oncol 2003; 22: 204.

34 Palmer $\mathrm{DH}$ et al. Virus directed enzyme prodrug therapy: intratumoural administration of a replication-deficient adenovirus encoding nitroreductase to patients with resectable liver cancer. J Clin Oncol 2004; 22: 1546-1552.

35 DeWeese TL et al. A phase I trial of CV706, a replicationcompetent, PSA selective oncolytic adenovirus, for the treatment of locally recurrent prostate cancer following radiation therapy. Cancer Res 2001; 61: 7464-7472.

36 Teh BS et al. Combining radiotherapy with gene therapy (from the bench to the bedside): a novel treatment strategy for prostate cancer. Oncologist 2002; 7: 458-466.

37 Hall SJ et al. Cooperative therapeutic effects of androgen ablation and adenovirus-mediated herpes simplex virus thymidine kinase gene and ganciclovir therapy in experimental prostate cancer. Cancer Gene Ther 1999; 6: 54-63. 